\title{
Deep Learning for Minimum Mean-Square Error Approaches to Speech Enhancement
}

\author{
Aaron Nicolson*, Kuldip K. Paliwal \\ Signal Processing Laboratory, Griffith University, Brisbane, Queensland, Australia, 4111
}

\begin{abstract}
Recently, the focus of speech enhancement research has shifted from minimum mean-square error (MMSE) approaches, like the MMSE short-time spectral amplitude (MMSE-STSA) estimator, to state-of-the-art masking-and mapping-based deep learning approaches. We aim to bridge the gap between these two differing speech enhancement approaches. Deep learning methods for MMSE approaches are investigated in this work, with the objective of producing intelligible enhanced speech at a high quality. Since the speech enhancement performance of an MMSE approach improves with the accuracy of the used a priori signal-to-noise ratio (SNR) estimator, a residual long short-term memory (ResLSTM) network is utilised here to accurately estimate the a priori SNR. MMSE approaches utilising the ResLSTM a priori SNR estimator are evaluated using subjective and objective measures of speech quality and intelligibility. The tested conditions include real-world non-stationary and coloured noise sources at multiple SNR levels. MMSE approaches utilising the proposed a priori SNR estimator are able to achieve higher enhanced speech quality and intelligibility scores than recent masking- and mapping-based deep learning approaches. The results presented in this work show that the performance of an MMSE approach to speech enhancement significantly increases when utilising deep learning.
\end{abstract}

Availability: The proposed a priori SNR estimator is available at: https://github.com/anicolson/DeepXi

Keywords: Speech enhancement; A priori SNR estimation; Minimum mean-square error (MMSE) approach; Residual long short-term memory (ResLSTM) network; Deep Xi; Speech separation.

\section{Introduction}

The minimum mean-square error short-time spectral amplitude (MMSE-STSA) estimator is the benchmark against which other speech enhancement methods are evaluated against [1]. Other prominent MMSE approaches to speech enhancement include the minimum mean-square error log-spectral amplitude (MMSE-LSA) estimator [2] and the Wiener filter (WF) approach [3]. While once at the forefront of speech enhancement research, less attention has been paid to the aforementioned MMSE approaches as of late. The research focus of the speech enhancement community has turned to deep learning methods.

Deep learning methods have recently been employed for speech enhancement, and have demonstrated state-ofthe-art performance [4]. Neural networks have been used as non-linear maps from noisy speech spectra to clean speech spectra. A denoising autoencoder (DAE) was pretrained for this task using noisy and clean speech pairs [5]. A non-causal neural network clean speech spectrum estimator was proposed that produced enhanced speech

\footnotetext{
* Corresponding author

Email addresses: aaron.nicolson@griffithuni.edu.au (Aaron Nicolson), k.paliwal@griffith.edu.au (Kuldip K. Paliwal)
}

with high objective quality scores [6], which later incorporated multi-objective learning and ideal binary mask (IBM)-based post-processing [7]. Neural networks have also been utilised to estimate time-frequency masks. A long short-term memory (LSTM) network was used recently to estimate the ideal ratio mask (IRM) [8].

We aim to bridge the gap between MMSE and deep learning approaches to speech enhancement, with the objective of producing enhanced speech that achieves higher quality and intelligibility scores than that of recent masking- and mapping-based deep learning approaches. Here, the performance improvement that deep learning methods can provide to the aforementioned MMSE approaches is investigated. Each MMSE approach requires the a priori signal-to-noise ratio (SNR) estimate of a noisy speech spectral component. The a priori SNR is formally described in Subsection 2.2. Since the performance of an MMSE approach to speech enhancement improves with the accuracy of the used a priori SNR estimator, deep learning methods are used here to accurately estimate the a priori SNR.

A priori SNR estimation is a difficult task, especially when considering the multitude of different noise sources. The decision-directed (DD) approach [1] to a priori SNR estimation was introduced with the MMSE-STSA estima- 
tor, and uses a weighted average of the a priori SNR estimate from the previous and current frames. The DD approach suffers from a frame delay problem [9], which is addressed by the two-step noise reduction (TSNR) technique [10]. Harmonic regeneration noise reduction (HRNR) [11] further improves upon the TSNR technique by computing an a priori SNR estimate from enhanced speech with artificially restored harmonics. Other a priori SNR estimates are computed using a maximum-likelihood approach. Selective cepstro-temporal smoothing (SCTS) [12] performs adaptive temporal smoothing on the cepstral representation of the maximum-likelihood estimate of the clean speech power spectrum, in order to estimate the a priori SNR.

It has been demonstrated that residual long shortterm memory (ResLSTM) networks are proficient acoustic models [13]. Motivated by this, a causal ResLSTM network, and a non-causal residual bidirectional LSTM (ResBLSTM) network [14] are used here for a priori SNR estimation. Unlike previous a priori SNR estimators, the proposed estimators do not require a noise estimator. Recently, a recurrent neural network (RNN) was used to aid the DD approach in a priori SNR estimation [15]. The proposed estimators differ by directly estimating the a priori SNR. This was accomplished by using the oracle case as the training target, where the oracle case is defined as the a priori SNR computed from the clean speech and noise. It was found that mapping the oracle a priori SNR target values to the interval $[0,1]$ improved the rate of convergence of the used stochastic gradient descent algorithm. We propose to use the cumulative distribution function (CDF) of the oracle a priori $\mathrm{SNR}$ in $\mathrm{dB}$ as the map. By using the $\mathrm{CDF}$, large sections of the distribution are not excluded.

In this work, MMSE approaches utilising deep learning are evaluated using subjective and objective measures of speech quality and intelligibility. The tested conditions include real-world non-stationary and coloured noise sources at multiple SNR levels. The MMSE approaches utilising deep learning are compared to recent maskingand mapping-based deep learning approaches to speech enhancement. Frame-wise spectral distortion (SD) levels are used to evaluate the accuracy of the proposed a priori SNR estimators. The speech enhancement performance of the mapped a priori SNR, the IRM, and the clean speech magnitude spectrum as the training target is also evaluated.

The paper is organised as follows: background knowledge is presented in Section 2, including the analysis, modification, and synthesis (AMS) procedure, a priori SNR, and MMSE approaches; the mapped a priori SNR training target is described in Section 3; the ResLSTM and ResBLSTM a priori SNR estimators are described in Section 4; the experiment setup is described in Section 5, including the objective and subjective testing procedures; the results and discussion are presented in Section 6; conclusions are drawn in Section 7.

\section{Background}

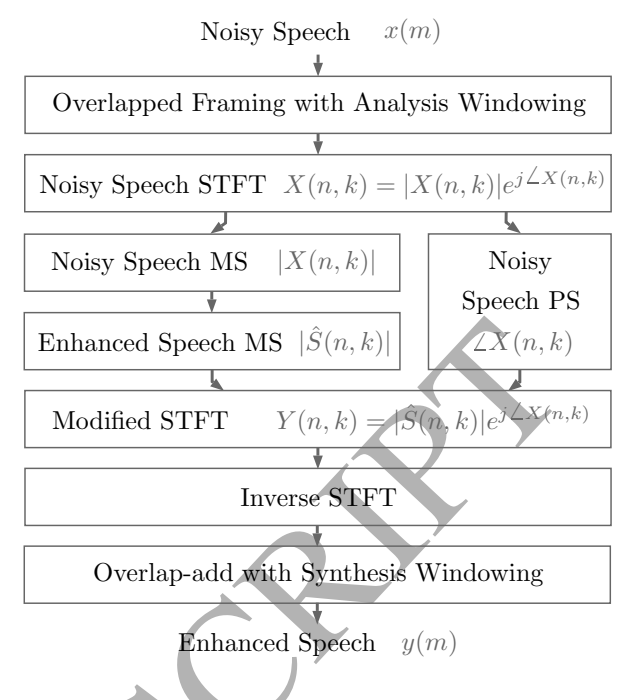

Figure 1: Block diagram of the short-time Fourier AMS speech enhancement framework.

\subsection{AMS Speech Enhancement Framework}

The short-time Fourier analysis, modification, and synthesis (AMS) framework was used to produce enhanced speech. The AMS framework [16, 17] consists of three stages: (1) the analysis stage, where noisy speech undergoes short-time Fourier transform (STFT) analysis; (2) the modification stage, where the noisy speech STFT is compensated for noise distortion to produce the modified STFT; and (3) the synthesis stage, where the inverse STFT operation is followed by overlap-add synthesis to construct the enhanced speech. A block diagram of the AMS framework is shown in Figure 1.

An uncorrelated additive noise model is assumed:

$$
x(m)=s(m)+d(m),
$$

where $x(m), s(m)$, and $d(m)$ denote the noisy speech, clean speech, and noise, respectively, and $m$ denotes the discretetime index. Noisy speech is analysed frame-wise using the running STFT [18]:

$$
X(n, k)=\sum_{m=0}^{N_{l}-1} x\left(m+n N_{s}\right) w(m) e^{-j 2 \pi m k / N_{l}},
$$

where $n$ denotes the frame index, $k$ denotes the discretefrequency index, $N_{l}$ denotes the frame length in discretetime samples, $N_{s}$ denotes the frame shift in discrete-time samples, and $w(m)$ is the analysis window function.

In polar form, the STFT of the noisy speech is expressed as

$$
X(n, k)=|X(n, k)| e^{j L X(n, k)}
$$


where $|X(n, k)|$ denotes the short-time noisy speech magnitude spectrum and $\angle X(n, k)$ denotes the short-time noisy speech phase spectrum. The noisy speech magnitude spectrum is enhanced, while the noisy speech phase spectrum remains unchanged. The enhanced speech magnitude spectrum is an estimate of the clean speech magnitude spectrum, and is denoted by $|\hat{S}(n, k)|$. The modified STFT is constructed by combining the enhanced speech magnitude spectrum with the noisy speech phase spectrum:

$$
Y(n, k)=|\hat{S}(n, k)| e^{j \angle X(n, k)} .
$$

The enhanced speech is constructed by applying the inverse STFT operation to the modified STFT, followed by least-squares overlap-add synthesis [19, 20]:

$$
y(m)=\frac{\sum_{n=-\infty}^{\infty} w\left(m-n N_{s}\right) y_{f}\left(n, m-n N_{s}\right)}{\sum_{n=-\infty}^{\infty} w^{2}\left(m-n N_{s}\right)}
$$

where $y_{f}\left(n, m-n N_{s}\right)$ is the framed enhanced speech, after the inverse STFT operation has been applied to the modified STFT.

\subsection{A Priori SNR}

MMSE approaches to speech enhancement require $a$ priori knowledge of the clean speech and noise. The true, unobserved SNR, or the a priori SNR of a noisy speech spectral component, is used for gain computation and is defined by

$$
\xi(n, k)=\frac{\lambda_{s}(n, k)}{\lambda_{d}(n, k)},
$$

where $\lambda_{s}(n, k)=\mathrm{E}\left\{|S(n, k)|^{2}\right\}$ is the variance of the clean speech spectral component, and $\lambda_{d}(n, k)=\mathrm{E}\left\{|D(n, k)|^{2}\right\}$ is the variance of the noise spectral component. As the clean speech and noise are unobserved during enhancement, the a priori SNR must be estimated from the observed noisy speech. When training a supervised learning algorithm to estimate the a priori SNR, clean speech and noise are given (the oracle case). As a result, the variance of the clean speech and noise spectral components are replaced by the squared magnitude of the clean speech and noise spectral components, respectively. The oracle case has been called the local a priori SNR previously [21].

\subsection{MMSE Approaches to Speech Enhancement}

The minimum mean-square error short-time spectral amplitude (MMSE-STSA) estimator [1] is the optimal MMSE clean speech magnitude spectrum estimator. It uses both the a priori and a posteriori SNR of a given noisy speech spectral component to compute the gain function. The a posteriori SNR is given by

$$
\gamma(n, k)=\frac{|X(n, k)|^{2}}{\lambda_{d}(n, k)} .
$$

The MMSE-STSA estimator gain function is given by

$$
\begin{gathered}
G_{\text {MMSe-STSA }}(n, k)=\frac{\sqrt{\pi}}{2} \frac{\sqrt{\nu(n, k)}}{\gamma(n, k)} \exp \left(\frac{-\nu(n, k)}{2}\right)((1+ \\
\left.\nu(n, k)) I_{0}\left(\frac{\nu(n, k)}{2}\right)+\nu(n, k) I_{1}\left(\frac{\nu(n, k)}{2}\right)\right)
\end{gathered}
$$

where $I_{0}(\cdot)$ and $I_{1}(\cdot)$ denote the modified Bessel functions of zero and first order, respectively, and $\nu(n, k)$ is given by

$$
\nu(n, k)=\frac{\xi(n, k)}{\xi(n, k)+1} \chi(n, k)
$$

The minimum mean-square error log-spectral amplitude (MMSE-LSA) estimator minimises the meansquared error between the clean and enhanced speech logmagnitude spectra [2]. The MMSE-LSA gain function is given by

$$
G_{\text {MMSe-LSA }}(n, k)=\frac{\xi(n, k)}{\xi(n, k)+1} \exp \left\{\frac{1}{2} \int_{\nu(n, k)}^{\infty} \frac{e^{-t}}{t} d t\right\} .
$$

The integral in Equation 10 is known as the exponential integral.

The Wiener filter (WF) approach to estimating the clean speech magnitude spectrum [3] minimises the meansquared error between the clean and enhanced speech complex discrete Fourier transform (DFT) coefficients. The gain function for the WF approach is given by

$$
G_{\mathrm{WF}}(n, k)=\frac{\xi(n, k)}{\xi(n, k)+1} .
$$

The recently popularised ideal ratio mask (IRM) [8] is the square-root WF (SRWF) approach gain function [22] computed from given clean speech and noise:

$$
G_{\text {SRWF }}(n, k)=\sqrt{\frac{\xi(n, k)}{\xi(n, k)+1}} .
$$

\section{Mapped A Priori SNR Training Target}

In preliminary experiments, it was found that mapping the oracle a priori SNR (in $\mathrm{dB}$ ) training target values for the $k^{\text {th }}$ noisy speech spectral component, $\xi_{\mathrm{dB}}(n, k)$, to the interval $[0,1]$ improved the rate of convergence of the used stochastic gradient descent algorithm. The cumulative distribution function $(\mathrm{CDF})$ of $\xi_{\mathrm{dB}}(n, k)$ was used as the map. It is assumed that $\xi_{\mathrm{dB}}(n, k)$ is distributed normally with mean $\mu_{k}$ and variance $\sigma_{k}^{2}: \xi_{\mathrm{dB}}(n, k) \sim \mathcal{N}\left(\mu_{k}, \sigma_{k}^{2}\right)$. Thus, the map is given by

$$
\bar{\xi}(n, k)=\frac{1}{2}\left[1+\operatorname{erf}\left(\frac{\xi_{\mathrm{dB}}(n, k)-\mu_{k}}{\sigma_{k} \sqrt{2}}\right)\right],
$$



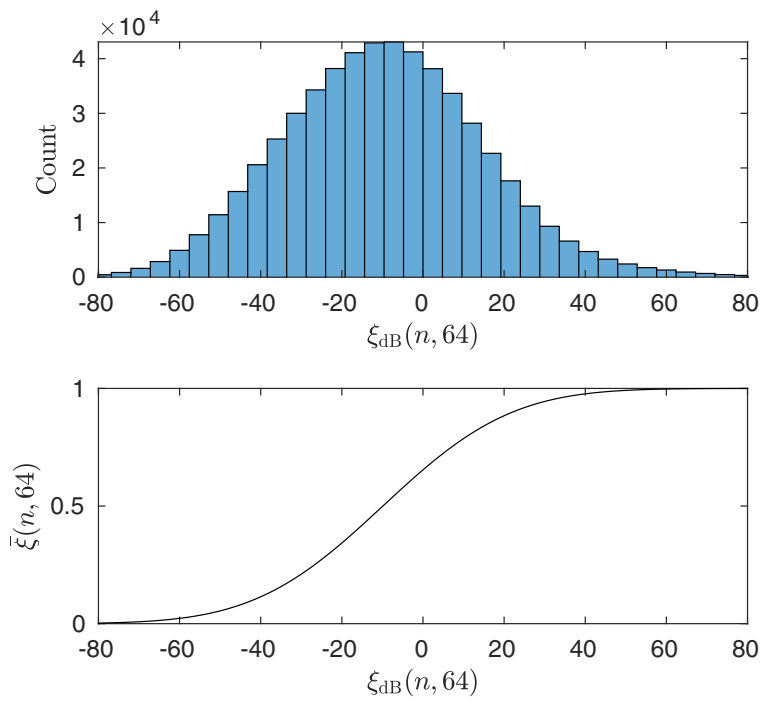

Figure 2: (Top) The distribution of $\xi_{\mathrm{dB}}(n, 64)$, over a sample of the training set. (Bottom) The CDF of $\xi_{\mathrm{dB}}(n, 64)$, assuming that $\xi_{\mathrm{dB}}(n, 64)$ is distributed normally (the sample mean and variance were found over the sample of the training set).

where $\bar{\xi}(n, k)$ is the mapped a priori SNR.

The statistics of $\xi_{\mathrm{dB}}(n, k)$ for the $k^{t h}$ noisy speech spectral component were found over a sample of the training set $^{1}$. As an example, the distribution of $\xi_{\mathrm{dB}}(n, 64)$ found over the aforementioned sample is shown in Figure 2 (top). It can be seen that it follows a normal distribution. A poorly chosen logistic map will force large sections of the distribution to the endpoints of the target interval, $[0,1$ ]. The CDF of $\xi_{\mathrm{dB}}(n, 64)$ over the sample is shown in Figure 2 (bottom), and is used to map the distribution of $\xi_{\mathrm{dB}}(n, 64)$ to the interval $[0,1]$.

\section{ResLSTM \& ResBLSTM A Priori SNR Esti- mators}

A residual long short-term memory (ResLSTM) network [13] is used to estimate the a priori SNR for the MMSE approaches, as showh in Figure 3. A ResLSTM consists of multiple residual blocks, with each block learning a residual function/ with reference to its input [23]. Residual connections allow for deep, powerful architectures [24]. The input to the ResLSTM is the magnitude spectrum of the $n^{t h}$ noisy speech frame, $|X(n, k)|$, for $k=$ $0,1, \ldots, N_{l} / 2$, where $N_{l}$ is the frame length in discrete-time

\footnotetext{
${ }^{1}$ The sample mean and variance of $\xi_{\mathrm{dB}}(n, k)$ for the $k^{t h}$ noisy speech spectral component were found over 1250 noisy speech signals created from the training clean speech and noise sets (Section 5). 250 randomly selected (without replacement) clean speech signals from the training clean speech set were mixed with random sections of randomly selected (without replacement) noise signals from the training noise set. Each of these were mixed at five different SNR levels: -5 to $15 \mathrm{~dB}$, in $5 \mathrm{~dB}$ increments.
}

samples. The ResLSTM estimates the a priori $\mathrm{SNR}^{2}$ for each of the noisy speech magnitude spectrum components.

The ResLSTM consists of 5 residual blocks, with each block containing a long short-term memory (LSTM) cell $[25,26], \mathbf{F}$, with a cell size of 512. LSTM cells are capable of learning both short and long-term temporal dependencies. Using LSTM cells within the residual blocks enables the ResLSTM to be a proficient sequence-based model. The residual connection is from the input of the residual block to after the LSTM cell activation [27]. FC is a fully-connected layer with 512 Rectified Linear Units (ReLUs) [28]. Layer normalisation is used before the activation function of FC [29]. The output/ayer, $\mathbf{O}$, is a fully-connected layer with sigmoidal units.

Shown in Figure 4 is the non-causal residual bidirectional long short-term memory (ResBLSTM) network $a$ priori SNR estimator. The ResBLSTM network is identical to the ResLSTM network, except that the residual blocks include both a forward and backward LSTM cell (F and B, respectively) [14], each with a cell size of 512 . While the concatenation of the forward and backward cell activations before the residual connection is standard for a ResBLSTM [30], the summation of the activations is used in this work ${ }^{3}$. This was to maintain a cell and residual connection size of 512, and to avoid the use of long shortterm memory projection (LSTMP) cells [32]. The residual connection was applied from the input of the residual block to after the summation of the forward and backward cell activations.

Details about the training strategy for the ResLSTM and ResBLSTM a priori SNR estimators are given in Subsection 5.3. Training time, memory usage, and speech enhancement performance were considered when selecting the hyperparameters for the ResLSTM and ResBLSTM networks ${ }^{4}$.

\section{Experiment Setup}

\subsection{Signal Processing, Noise Estimation, and a posteriori SNR estimation}

The Hamming window function was used for analysis and synthesis [33, 34, 35], with a frame length of $32 \mathrm{~ms}$ $\left(N_{l}=512\right)$ and a frame shift of $16 \mathrm{~ms}\left(N_{s}=256\right)$. The a priori SNR was estimated from the 257-point singlesided noisy speech magnitude spectrum, which included

\footnotetext{
${ }^{2} \hat{\xi}(n, k)$ values are obtained by applying the inverse of Equation $13\left(\hat{\xi}_{\mathrm{dB}}(n, k)=\sigma_{k} \sqrt{2} \operatorname{erf}^{-1}(2 \hat{\bar{\xi}}(n, k)-1)+\mu_{k}\right)$, followed by $10\left(\hat{\xi}_{\mathrm{dB}}(n, k) / 10\right)$ to the $\hat{\bar{\xi}}(n, k)$ values.

${ }^{3}$ Following the intuition that residual networks behave like ensembles of relatively shallow networks [31], the summation of the forward and backward activations can be viewed as an ensemble of the activations with no weighting.

${ }^{4}$ The time taken for the completion of one training epoch for the ResLSTM and the ResBLSTM networks was approximately 9 and 18 hours, respectively (NVIDIA GTX 1080 Ti GPUs were used).
} 


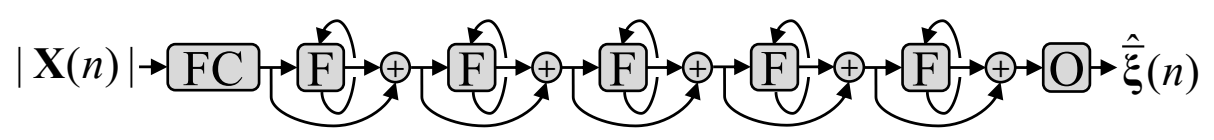

Figure 3: ResLSTM a priori SNR estimator. FC is a fully-connected layer. The output layer, $\mathbf{O}$, is a fully-connected output layer with sigmoidal units. Each residual block contains an LSTM cell, F.

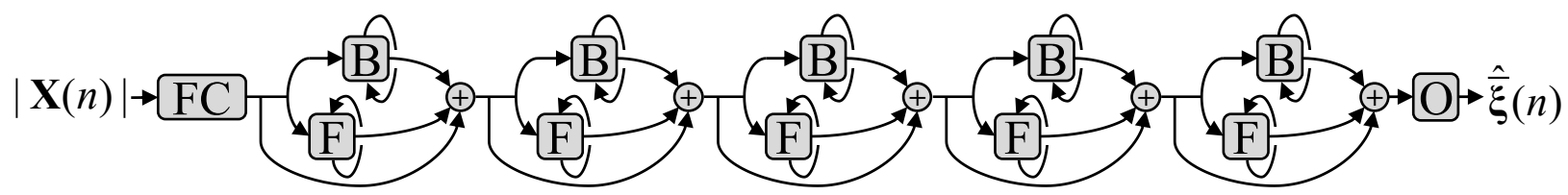

Figure 4: ResBLSTM a priori SNR estimator. FC is a fully-connected layer. The output layer, $\mathbf{O}$, is a fully-connected layer with sigmoidal units. Each residual block contains a forward and backward LSTM cell, F and B, respectively.

both the DC frequency component and the Nyquist frequency component. The MMSE-based noise estimator with speech presence probability (SPP) from [36] was used by the DD, TSNR, HRNR, and SCTS a priori SNR estimation methods. The a posteriori SNR was estimated using both the observed noisy speech and the noise estimator when the DD approach, TSNR, HRNR, and SCTS a priori SNR estimation methods were used. When the ResLSTM and ResBLSTM a priori SNR estimators were used, the $a$ posteriori SNR was estimated from the a priori SNR estimate using the following relationship: $\hat{\gamma}(n, k)=\hat{\xi}(n, k)+1$.

\subsection{Training Set}

The train-clean-100 set from the Librispeech corpus [37] (28539 utterances), the CSTR VCTK Corpus [38] (42 015 utterances), and the $s i^{*}$ and $s x^{*}$ training sets from the TIMIT corpus [39] (3696 utterances) were included in the clean speech training set. The QUT-NOISE dataset [40], the Nonspeech dataset [41], the Environmental Background Noise dataset $[42,43]$, the noise set from the MUSAN corpus [44], multiple FreeSound packs ${ }^{5}$, and coloured noise recordings (with an $\alpha$ value ranging from -2 to 2 in increments of 0.25 ) were included in the noise training set (2 382 recordings). All clean speech and noise signals were single-channel, with a sampling frequency of $16 \mathrm{kHz}$. The noise corruption procedure for the training set is described in Subsection 5.3.

\subsection{Training Strategy}

The following strategy was employed for neural network training:

- Cross-entropy as the loss function.

- The Adam algorithm [45] for gradient descent optimisation.

\footnotetext{
${ }^{5}$ Freesound packs that were used: 147, 199, 247, 379, 622, 643 , 1 133, 1 563, 1 840, 2 432, 4 366, 4 439, 15 046, 15 598, 21558.
}

- $5 \%$ of the clean speech training set was used as a validation set.

- For each mini-batch, each clean speech signal was mixed with a random section of a randomly selected noise signal from the noise training set at a randomly selected SNR level ( -10 to $20 \mathrm{~dB}$, in $1 \mathrm{~dB}$ increments) to create the noisy speech signals.

- A mini-batch size of 10 noisy speech signals.

- The selection order for the clean speech signals was randomised before each epoch.

- A total of 10 epochs were used to train the ResLSTM and ResBLSTM networks.

- The LSTM-IRM estimator from [8] was replicated here, and used the noisy speech magnitude spectrum (as described in Subsection 5.1) as its input. It was trained for 10 epochs using the aforementioned training set.

\subsection{Test Set}

Four recordings of four real-world noise sources, including two non-stationary and two coloured, were included in the test set. The two real-world non-stationary noise sources included voice babble from the RSG-10 noise dataset [46] and street music ${ }^{6}$ from the Urban Sound dataset [47]. The two real-world coloured noise sources included F16 and factory (welding) from the RSG-10 noise dataset [46]. 10 clean speech signals were randomly selected (without replacement) from the TSP speech corpus ${ }^{7}$ [48] for each of the four noise signal. To create the noisy speech, a random section of the noise signal was mixed with the clean speech at the following SNR levels: -5 to $15 \mathrm{~dB}$, in $5 \mathrm{~dB}$ increments. This created a test set of 200 noisy speech files. The noisy speech signals were single channel, with a sampling frequency of $16 \mathrm{kHz}$.

\footnotetext{
${ }^{6}$ Street music recording number 26270 was used from the Urban Sound dataset.

${ }^{7}$ Only adult speakers were included from the TSP speech corpus.
} 


\subsection{Spectral Distortion}

The frame-wise spectral distortion (SD) [49] is defined as the root-mean-square difference between the a priori SNR estimate in $\mathrm{dB}, \hat{\xi}_{\mathrm{dB}}(n, k)$, and the oracle case in $\mathrm{dB}$, $\xi_{\mathrm{dB}}(n, k)$, for the $n^{\text {th }}$ frame $^{8}$ :

$$
D_{n}^{2}=\frac{1}{N_{l} / 2+1} \sum_{k=0}^{N_{l} / 2}\left[\xi_{\mathrm{dB}}(n, k)-\hat{\xi}_{\mathrm{dB}}(n, k)\right]^{2} .
$$

Average SD levels were obtained over the test set.

\subsection{Objective Evaluation}

Objective measures were used to evaluate both the quality and intelligibility of the enhanced speech. Each objective measure evaluated the enhanced speech with respect to the corresponding clean speech. Average objective scores were obtained over the test set. The objective measures that were used included:

- The mean opinion score of the objective listening quality (MOS-LQO) [50] was used for objective quality evaluation, where the wideband perceptual evaluation of quality (Wideband PESQ) [51] was the objective model used to obtain the MOS-LQO.

- The short-time objective intelligibility (STOI) measure was used for objective intelligibility evaluation $[52,53]$.

\subsection{Subjective Evaluation}

Subjective testing was used to evaluate the quality of the enhanced speech produced by the speech enhancement methods. The mean subjective preference (\%) was used as the subjective quality measure. Mean subjective preference (\%) scores were determined from a series of AB listening tests [54]. Each AB listening test involved a stimuli pair. Each stimulus was either clean, noisy, or enhanced speech. The enhanced speech stimuli were produced by the MMSE-LSA estimator utilising the DD approach, Xu2017 [6,7], and the MMSE-LSA estimator utilising the ResBLSTM a priori SNR estimator. Therefore, each stimulus belonged to one of the following classes: clean speech, noisy speech, enhanced speech produced by the MMSE-LSA estimator utilising the DD approach, Xu2017 enhanced speech, or enhanced speech produced by the MMSE-LSA estimator utilising the ResBLSTM a priori SNR estimator.

After listening to a stimuli pair, the listeners' preference was determined by selecting one of three options. The first and second options indicated a preference for one of the two stimuli, while the third option indicated an equal preference for both stimuli. Pair-wise scoring was used, with a score of +1 awarded to the preferred class, and 0 to

\footnotetext{
${ }^{8} \xi_{d B}(n, k)$ and $\hat{\xi}_{d B}(n, k)$ values that were less than $-40 \mathrm{~dB}$, or greater than $60 \mathrm{~dB}$ were clipped to $-40 \mathrm{~dB}$ and $60 \mathrm{~dB}$, respectively.
}

Table 1: A priori SNR estimation SD levels for each of the a priori SNR estimators. The lowest SD for each noise source and at each SNR level is shown in boldface. The tested conditions include realworld non-stationary (voice babble and street music) and coloured (F16 and factory) noise sources at multiple SNR levels.

\begin{tabular}{|c|c|c|c|c|c|c|}
\hline \multirow[b]{2}{*}{ Noise } & \multirow[b]{2}{*}{$\hat{\xi}(n, k)$} & \multicolumn{5}{|c|}{ SNR level (dB) } \\
\hline & & -5 & 0 & 5 & 10 & 15 \\
\hline \multirow{6}{*}{$\begin{array}{l}\frac{0}{0} \\
0 \\
0 \\
0 \\
0 \\
0 \\
0 \\
0 \\
0\end{array}$} & $\mathrm{DD}[1]$ & 18.5 & 17.7 & 17.2 & 17.0 & 17.2 \\
\hline & TSNR[10] & 18.4 & 17.5 & 17.0 & 16.9 & 17.1 \\
\hline & HRNR[11] & 19.5 & 18.9 & 18.5 & 18.4 & 18.6 \\
\hline & $\operatorname{SCTS}[12]$ & 17.5 & 16.8 & 16.5 & 16.5 & 16.9 \\
\hline & ResLSTM & 14.5 & 13.9 & 13.3 & 12.8 & 12.4 \\
\hline & ResBLSTM & 12.7 & 12.1 & 11.6 & 11.2 & 10.9 \\
\hline \multirow{6}{*}{  } & $\mathrm{DD}[1]$ & 19.9 & & 17.6 & 17.0 & 16.8 \\
\hline & TSNR[10] & 19.7 & 18.4 & 17.4 & 16.8 & 16.6 \\
\hline & $\operatorname{HRNR}[11]$ & 19.8 & 18.7 & 17.9 & 17.5 & 17.5 \\
\hline & SCTS $[12]$ & 18.6 & 17. & 16.6 & 16.2 & 16.2 \\
\hline & ResLSTM & 13.5 & 13.1 & 12.7 & 12.3 & 12.0 \\
\hline & ResBLSTN & 11.8 & 11.4 & 11.1 & 10.7 & 10.5 \\
\hline \multirow{12}{*}{$\stackrel{0}{\vec{I}}$} & $\mathrm{DD}[1]$ & 22.1 & 20.5 & 19.2 & 18.2 & 17.5 \\
\hline & TSNR[10] & 21.8 & 20.2 & 18.9 & 17.9 & 17.2 \\
\hline & $\operatorname{HRNR}[11]$ & 20.7 & 19.4 & 18.4 & 17.7 & 17.3 \\
\hline & SCTS $[12]$ & 20.8 & 19.2 & 18.0 & 17.1 & 16.6 \\
\hline & ResLSTM & 13.3 & 12.7 & 12.3 & 12.0 & 11.7 \\
\hline & ResBLSTM & 11.5 & 11.0 & 10.7 & 10.4 & 10.2 \\
\hline & & 24.0 & 22.2 & 20.7 & 19.4 & 18.5 \\
\hline & TSNR $[10]$ & 23.7 & 22.0 & 20.4 & 19.2 & 18.3 \\
\hline & HRNR[11] & 23.0 & 21.4 & 20.1 & 19.1 & 18.4 \\
\hline & SCTS[12] & 22.4 & 20.7 & 19.3 & 18.2 & 17.4 \\
\hline & ResLSTM & 13.8 & 13.2 & 12.7 & 12.4 & 12.1 \\
\hline & ResBLSTM & 13.0 & 12.2 & 11.7 & 11.3 & 11.0 \\
\hline
\end{tabular}

the other. If the listener had an equal preference for both stimuli, each class was awarded a score of +0.5 . Participants could re-listen to the stimuli pair before selecting an option.

Two utterances ${ }^{9}$ from the test set were used as the clean speech stimuli: utterance $35 \_10$, as uttered by male speaker $M F$, and utterance 01_03, as uttered by female speaker $F A$. Voice babble from the test set was mixed with the clean speech stimuli at an SNR level of $5 \mathrm{~dB}$, producing the noisy speech stimuli. The enhanced speech stimuli for each of the speech enhancement methods was produced from the noisy speech stimuli. For each utterance, all possible stimuli pair combinations were presented to the listener (i.e. double-blind testing). Each participant listened to a total of 40 stimuli pair combinations. A total of five English-speaking listeners participated. Each listening test was conducted in a separate session, in a quiet room using closed circumaural headphones at a comfortable listening level.

\section{Results and Discussion}

\subsection{A Priori SNR Estimation Accuracy}

The a priori SNR estimation SD levels for each of the $a$ priori SNR estimators is shown in Table 1. The SD levels

\footnotetext{
${ }^{9}$ Using the entirety of the test set was not feasible.
} 
are used to evaluate the accuracy of each a priori SNR estimator. For real-world non-stationary noise sources, the ResLSTM a priori SNR estimator produced lower SD levels than the previous a priori SNR estimation methods (DD, TSNR, HRNR, and SCTS), with an average SD reduction of $4.7 \mathrm{~dB}$ when compared to the DD approach. The ResBLSTM a priori SNR estimator achieved an average SD reduction of $6.4 \mathrm{~dB}$ when compared to the DD approach, showing improved accuracy when causality is not a requirement. The proposed a priori SNR estimators also produced the lowest SD levels for the real-world coloured noise sources. The ResLSTM and ResBLSTM $a$ priori SNR estimators achieved an average SD reduction of 7.6 and $8.9 \mathrm{~dB}$, respectively, when compared to the DD approach.

The proposed a priori SNR estimators significantly outperform the previous a priori SNR estimation methods. Evaluating the results in [15], the RNN-assisted DD approach (a deep learing-based a priori SNR estimator) could only outperform the DD approach at higher SNR levels ( $5 \mathrm{~dB}$ and greater for signal-to-distortion ratio (SDR)). Here, the ResLSTM and ResBLSTM a priori SNR estimators significantly outperform the DD approach for all conditions.

\subsection{MMSE Approaches Utilising Deep Learning \\ 6.2.1. MMSE-STSA Estimator Utilising Deep Learning}

The objective quality and intelligibility scores for the MMSE-STSA estimator utilising each of the a priori SNR estimators are shown in Figures 5 and 6 , respectively. The MMSE-STSA estimator achieved the highest objective quality scores when deep learning was used, for both the real-world non-stationary and coloured noise sources. The MMSE-STSA estimator utilising the ResLSTM and ResBLSTM a priori SNR estimators achieved an average MOS-LQO improvement of 0.30 and 0.52 , respectively, compared to when the DD approach was used. The highest objective intelligibility scores were achieved by the MMSESTSA estimator when deep learning was used, for both the real-world non-stationary and coloured noise sources. The MMSE-STSA estimator utilising the ResLSTM and ResBLSTM a priori SNR estimators achieved an average STOI improvement of $5.8 \%$ and $8.2 \%$, respectively, compared to when the DD approach was used. The MMSESTSA estimator utilising either of the proposed a priori SNR estimators achieved higher objective intelligibility scores than noisy speech, a feat that it struggled to achieve consistently with the other a priori SNR estimation methods. It can be seen that there is a correlation between $a$ priori SNR estimation accuracy (given by the SD levels) and speech enhancement performance (given by the objective quality and intelligibility scores).

\subsubsection{MMSE-LSA Estimator Utilising Deep Learning}

The objective quality and intelligibility scores for the MMSE-LSA estimator utilising each of the a priori SNR estimators are shown in Figures 7 and 8, respectively. The MMSE-LSA estimator achieved the highest objective quality scores when deep learning was used, for both the real-world non-stationary and coloured noise sources. The MMSE-LSA estimator utilising the ResLSTM and ResBLSTM a priori SNR estimators achieved an average MOS-LQO improvement of 0.23 and 0.45 , respectively, compared to when the DD approach was used. The objective intelligibility scores show that deep learning enabled the MMSE-LSA estimator to produce the most intelligible enhanced speech, for both the real-world non-stationary and coloured noise sources. The MMSE-LSA estimator utilising the ResLSTM and ResBLSTM a priori SNR estimators achieved an average STOI improvement of $5.8 \%$ and $8.3 \%$, respectively, compared to when the DD approach was used.

\subsubsection{WF Approach Utilising Deep Learning}

The objective quality and intelligibility scores for the WF approach utilising each of the a priori SNR estimators are shown in Figures 9 and 10, respectively. The WF approach achieved the highest objective quality scores when deep learning was used, for both the real-world nonstationary and coloured noise sources. The WF approach utilising the ResLSTM and ResBLSTM a priori SNR estimators achieved an average MOS-LQO improvement of 0.13 and 0.32 , respectively, compared to when the DD approach was used. The objective intelligibility scores show that deep learning enabled the WF approach to produce the most intelligible enhanced speech, for both the realworld non-stationary and coloured noise sources. The WF approach utilising the ResLSTM and ResBLSTM a priori SNR estimators achieved an average STOI improvement of $5.5 \%$ and $8.5 \%$, respectively, compared to when the DD approach was used.

Table 2: The average improvement over the MMSE approach in the preceding row is shown for both objective quality (MOS-LQO) and intelligibility (STOI).

\begin{tabular}{llll}
\hline$\hat{\boldsymbol{\xi}}$ & Gain & MOS-LQO & STOI \\
\hline \multirow{3}{*}{ ResLSTM } & WF & - & - \\
& MMSE-STSA & +0.10 & $+1.76 \%$ \\
& MMSE-LSA & +0.02 & $-0.15 \%$ \\
\hline \multirow{3}{*}{ ResBLSTM } & WF & +0.07 & $+1.37 \%$ \\
& MMSE-STSA & +0.13 & $+1.19 \%$ \\
& MMSE-LSA & +0.02 & $-0.08 \%$ \\
\hline
\end{tabular}

\subsubsection{Comparison of MMSE approaches}

A comparison of each MMSE approach utilising the proposed a priori SNR estimators is shown in Table 2 . It can be seen that both the MMSE-STSA and MMSE-LSA estimators outperformed the WF approach. As described previously, the MMSE-STSA and MMSE-LSA estimators are optimal MMSE clean speech magnitude spectrum esti- 

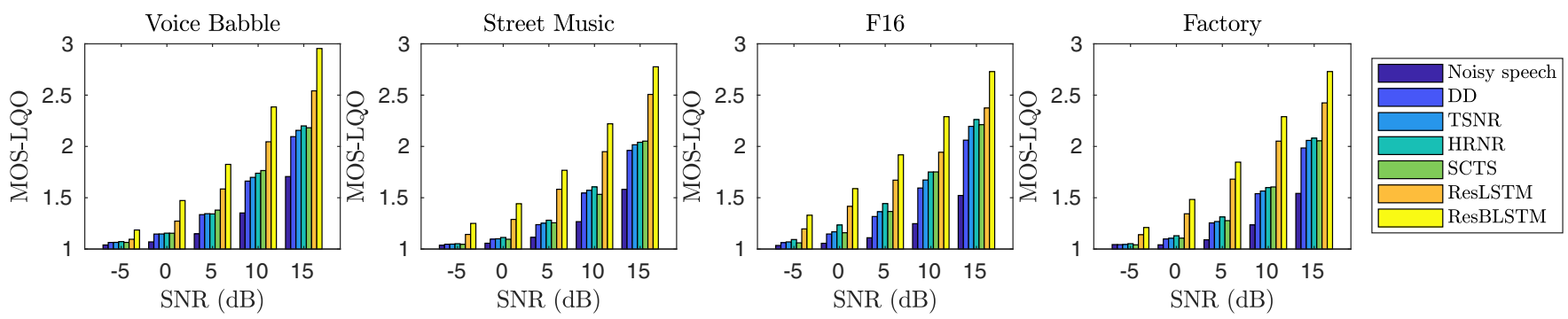

Figure 5: MMSE-STSA estimator objective quality (MOS-LQO) scores for each a priori SNR estimator. The tested conditions include real-world non-stationary (voice babble and street music) and coloured (F16 and factory) noise sources at multiple SNR levels.
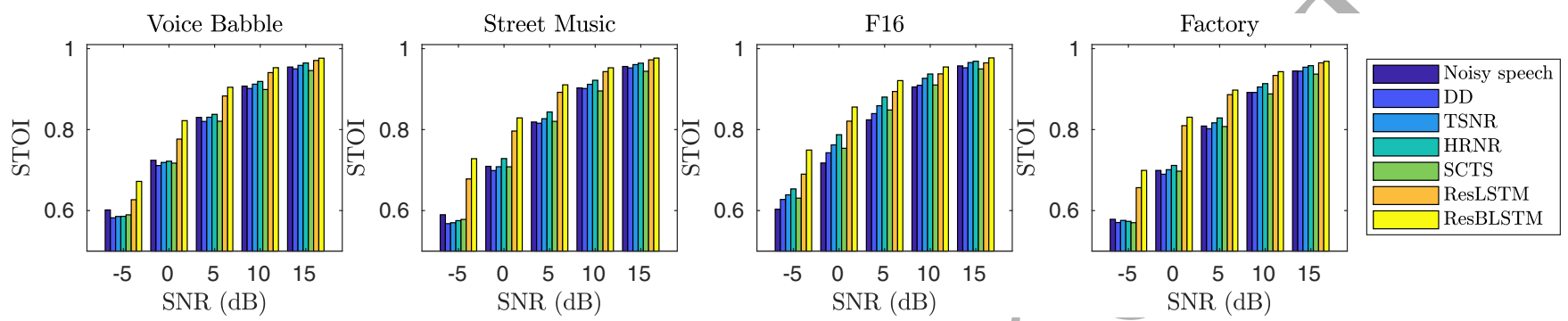

Figure 6: MMSE-STSA estimator objective intelligibility (STOI) scores for each a priori SNR estimator. The tested conditions include real-world non-stationary (voice babble and street music) and coloured (F16 and factory) noise sources at multiple SNR levels.
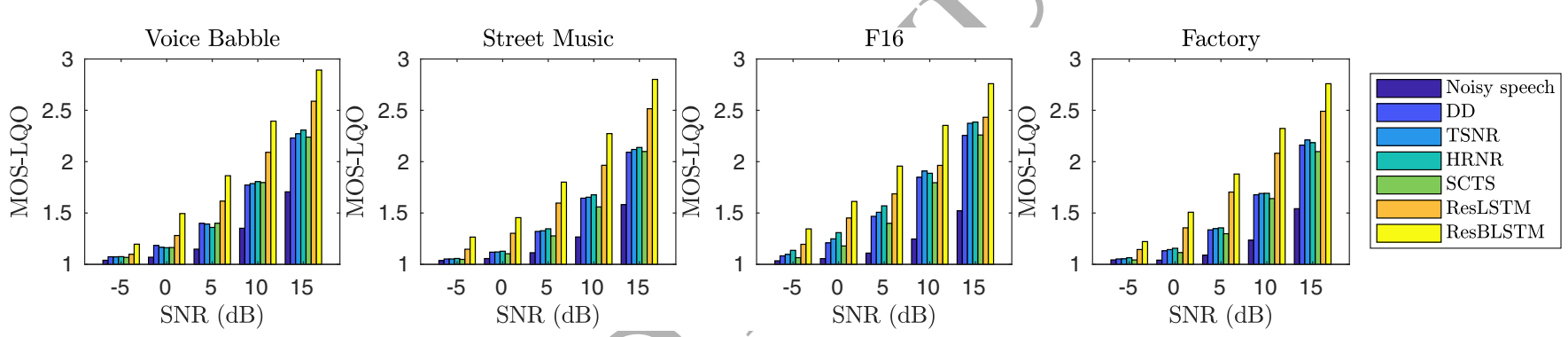

Figure 7: MMSE-LSA estimator objective quality (MOS-LQO) scores for each a priori SNR estimator. The tested conditions include real-world non-stationary (voice babble and street music) and coloured (F16 and factory) noise sources at multiple SNR levels.


Figure 8: MMSE-LSA estimator objective intelligibility (STOI) scores for each a priori SNR estimator. The tested conditions include real-world non-stationary (voice babble and street music) and coloured (F16 and factory) noise sources at multiple SNR levels.

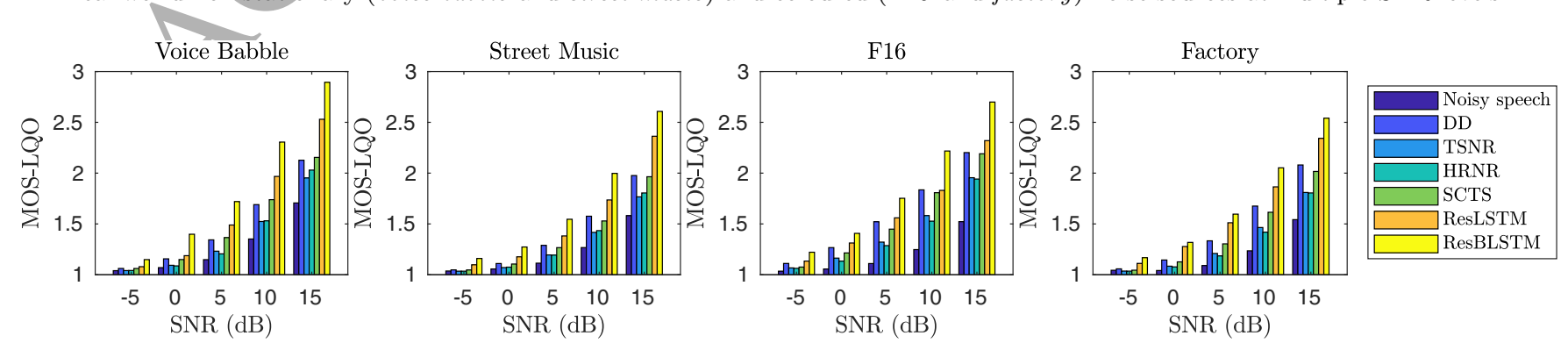

Figure 9: WF approach objective quality (MOS-LQO) scores for each a priori SNR estimator. The tested conditions include real-world non-stationary (voice babble and street music) and coloured (F16 and factory) noise sources at multiple SNR levels. 

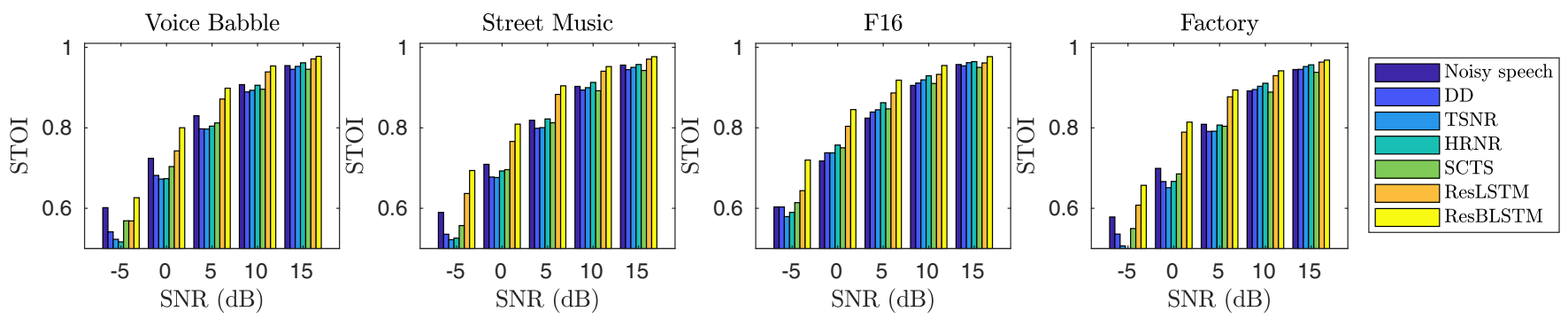

Figure 10: WF approach objective intelligibility (STOI) scores for each a priori SNR estimator. The tested conditions include real-world non-stationary (voice babble and street music) and coloured (F16 and factory) noise sources at multiple SNR levels.

mators $^{10}$, whereas the WF approach is the optimal MMSE clean speech complex DFT coefficient estimator. The target in this work is the clean speech magnitude spectrum, which favours the MMSE-STSA and MMSE-LSA estimators. This gives reason as to why the MMSE-STSA and MMSE-LSA estimators outperformed the WF approach. The MMSE-LSA estimator was selected for the speech enhancement comparison in Subsection 6.4 as it achieved the highest average objective quality score, and the second highest average objective intelligibility score.

Table 3: The average improvement over the training target in the preceding row is shown for both objective quality (MOS-LQO) and intelligibility (STOI).

\begin{tabular}{lll}
\hline Target & MOS-LQO & STOI \\
\hline$|S|$ & - & - \\
IRM & +0.33 & $+3.49 \%$ \\
$\bar{\xi}$ SRWF & +0.01 & $-0.89 \%$ \\
$\bar{\xi}$ MMSE-STSA & +0.03 & $-0.09 \%$ \\
$\bar{\xi}$ MMSE-LSA & +0.02 & $-0.15 \%$ \\
\hline
\end{tabular}

\subsection{Comparison of Training Targets}

Here, the speech enhancement performance of the (mapped) a priori SNR, the IRM, and the clean speech magnitude spectrum as the training target is evaluated. The training strategy described in Subsection 5.3 was used to train an identical ResLSTM network for each training target ${ }^{11}$. The SRWF approach, MMSE-STSA estimator, and the MMSE-LSA estimator are used to evaluate the $a$ priori SNR training target. The SRWF approach is used instead of the WF approach as it has the same form as the IRM. The objective quality and intelligibility scores achieved by each training target are shown in Figures 11 and 12, respectively. The a priori SNR training target achieved the highest objective quality scores, for both the real-world non-stationary and coloured noise sources (except for voice babble at $15 \mathrm{~dB}$ ). However, the IRM training

\footnotetext{
${ }^{10}$ Specifically, the MMSE-LSA estimator is the optimal clean speech log-magnitude spectrum estimator.

${ }^{11}$ The cross-entropy loss function was used when optimising for the mapped a priori SNR and the IRM. In contrast, the quadratic loss function was used when optimising for the clean speech MS, as its values are not bounded to the interval $[0,1]$.
}

target achieved the highest objective intelligibility scores, for both the real-world non-stationary and coloured noise sources (except for factory at $0 \mathrm{~dB}$ ).

It can be seen in Table 3 and in Figures 11 and 12 that the a priori SNR and the IRM both outperform the clean speech magnitude spectrum as the training target. These results are consistent with those reported in the literature. A study on training targets by Wang et al. [55] found that the IRM as the training target produces significantly higher objective quality and intelligibility scores than the clean speech magnitude spectrum (as indicated by FFT-MAG in [55]) for both real-world non-stationary and coloured noise sources at multiple SNR levels $(-5,0$, and $5 \mathrm{~dB}$ ). It has also been shown by Zhao et al. [56] that higher objective intelligibility scores are obtained when the IRM is used instead of the clean speech magnitude spectrum as the training target, for voice babble at multiple SNR levels $(-5,0$, and $5 \mathrm{~dB}$ ) (as shown by Figure 2 in [55]).

As can be seen in Table 3, there is a trade-off between enhanced speech quality and intelligibility when selecting between the IRM and the a priori SNR as the training target. If it is desired to produce enhanced speech that is more intelligible, the IRM should be chosen as the training target. If it is desired for the enhanced speech to have a higher quality, the a priori SNR should be chosen as the training target. A further trade-off between enhanced speech quality and intelligibility can be made through the selection of the MMSE approach. Amongst the MMSE approaches, the SRWF approach produces the most intelligible enhanced speech, but with the worst quality. On the contrary, the MMSE-LSA estimator produces the least intelligible enhanced speech, but with the highest quality. The MMSE-STSA estimator offers a compromise between the SRWF approach and the MMSE-LSA estimator.

\subsection{Comparison of Speech Enhancement Methods}

Here, an MMSE approach utilising deep learning is compared to both a masking- and a mapping-based deep learning approach to speech enhancement. The MMSELSA estimator, utilising the ResLSTM and ResBLSTM a priori SNR estimators, is compared to the LSTMIRM estimator from [8], and the non-causal neural net- 

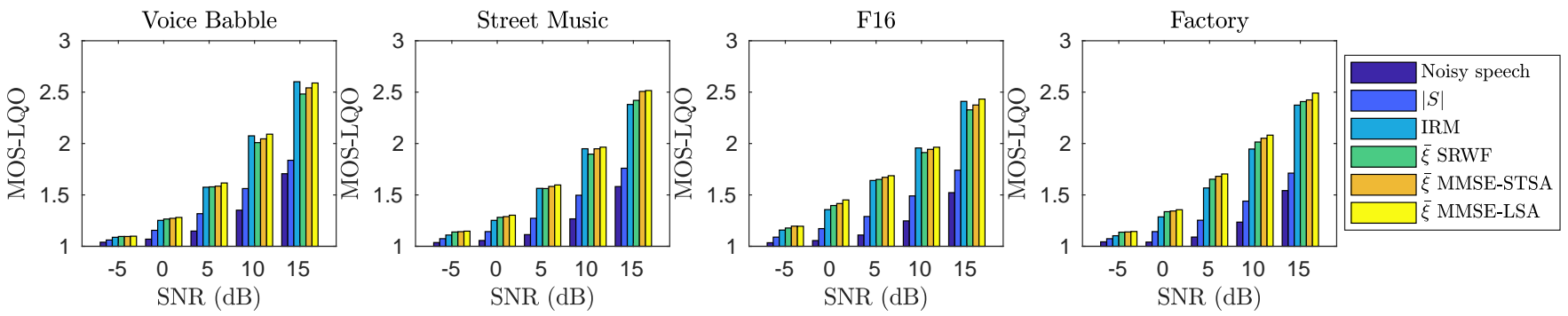

Figure 11: Objective quality (MOS-LQO) scores for each training target. The tested conditions include real-world non-stationary (voice babble and street music) and coloured (F16 and factory) noise sources at multiple SNR levels.
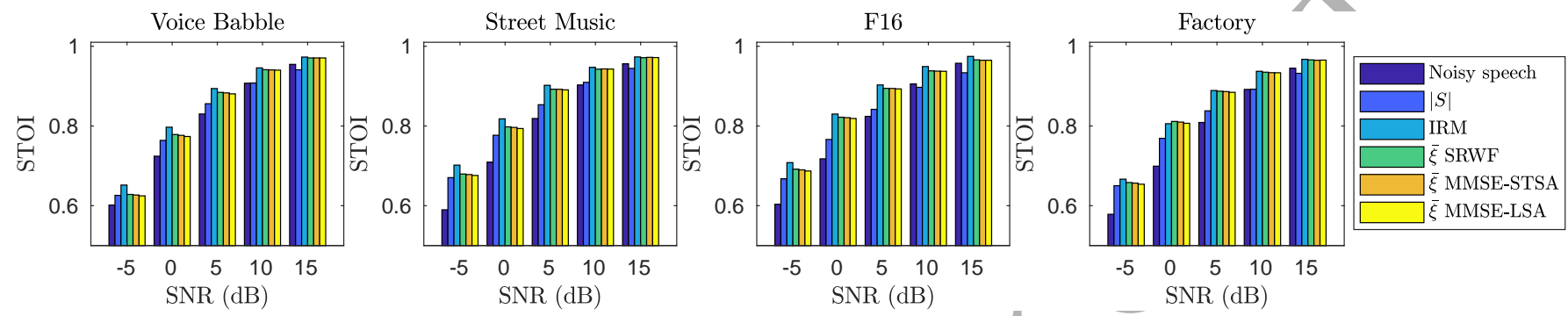

Figure 12: Objective intelligibility (STOI) scores for each training target. The tested conditions include real-world non-stationary (voice babble and street music) and coloured (F16 and factory) noise sources at multiple SNR levels.

work clean speech spectrum estimator ${ }^{12}$ that uses multiobjective learning and IBM-based post-processing from $[6,7]$, referred to as Xu2017 in this subsection. The MMSE-LSA estimator utilising the DD approach is also compared, to represent earlier speech enhancement methods.

\subsubsection{Objective Scores}

The objective quality and intelligibility seores achieved by each of the speech enhancement methods for each tested condition are shown in Figures 13 and 14, respectively. The MMSE-LSA estimator utilising the non-causal ResBLSTM a priori SNR estimator produced enhanced speech with higher objective quality and intelligibility scores than the LSTM-IRM estimator and Xu2017 for both real-world non-stationary and coloured noise sources. The MMSE-LSA estimator utilising the causal ResLSTM a priori SNR estimator achieved higher objective intelligibility scores than Xu2017 for all conditions, and the LSTM-IRM estimator for all noise sources other than voice babble. It also achieved higher objective quality scores than the LSTM-IRM estimator for all conditions, and Xu2017 for street music at high SNR levels, for F16 at low SNR levels, and for factory at all SNR levels. It is important to stress that Xu2017 is a non-causal system, whilst the ResLSTM a priori SNR estimator is a causal system.

Table 4 details the average improvement that the proposed a priori SNR estimators hold over the other speech enhancement methods. The MMSE-LSA estimator utilising the causal ResLSTM a priori SNR estimator achieved

\footnotetext{
${ }^{12}$ Five past and five future frames are used as part of its input feature vector.
}

Table 4: The average improvement over the speech enhancement method in the preceding row is shown for both objective quality (MOS-LQO) and intelligibility (STOI).

\begin{tabular}{llll}
\hline \multicolumn{1}{c}{ Method } & Casual & MOS-LQO & STOI \\
\hline MMSE-LSA; DD $\hat{\xi}[1,36]$ & Yes & - & - \\
LSTM-IRM est.[8] & Yes & +0.01 & $+4.52 \%$ \\
MMSE-LSA; ResLSTM $\hat{\xi}$ & Yes & +0.22 & $+1.28 \%$ \\
\hline Xu2017[6, 7] & No & +0.01 & $-2.59 \%$ \\
MMSE-LSA; ResBLSTM $\hat{\xi}$ & No & +0.21 & $+5.08 \%$ \\
\hline
\end{tabular}

the highest average objective quality and intelligibility scores amongst the causal speech enhancement methods. It also achieved a higher average intelligibility score than Xu2017 (a non-causal system). The MMSE-LSA estimator utilising the non-causal ResBLSTM a priori SNR estimator achieved the highest average objective quality and intelligibility scores amongst all the speech enhancement methods.

The advantages and disadvantages of each deep learning approach to speech enhancement can be seen in Table 4, as well as Figures 13 and 14. The advantage of Xu2017 is that it can produce enhanced speech with high objective quality scores. However, it produces enhanced speech with low objective intelligibility scores. The reverse is true for the LSTM-IRM estimator. It produces enhanced speech with low objective quality scores, but high objective intelligibility scores. On the other hand, the MMSE-LSA estimator utilising the proposed a priori SNR estimators is able to produce enhanced speech with both high objective quality and intelligibility scores.

When considering the training target results from Sub- 

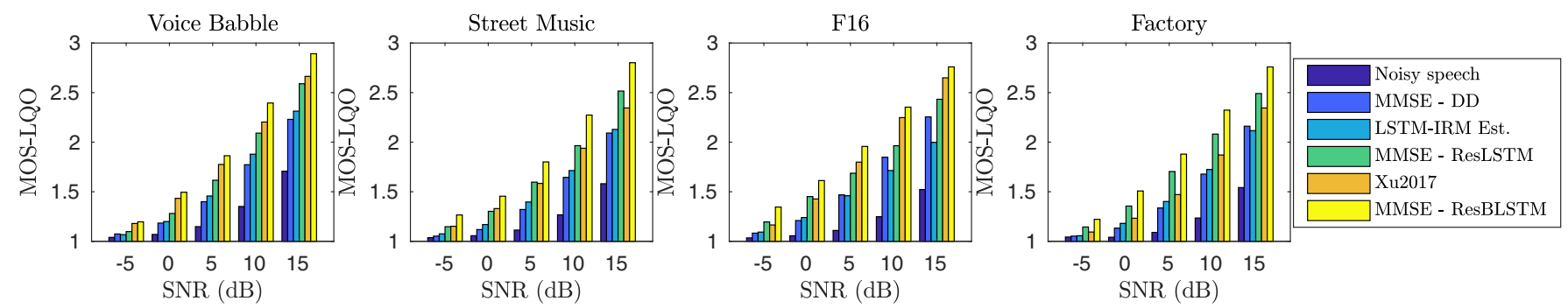

Figure 13: Objective quality (MOS-LQO) scores for the MMSE-LSA estimator utilising the DD approach, the LSTM-IRM estimator, Xu2017, and the MMSE-LSA estimator utilising both the ResLSTM and ResBLSTM a priori SNR estimators. The tested conditions include real-world non-stationary (voice babble and street music) and coloured (F16 and factory) noise sources at multiple SNR levels.
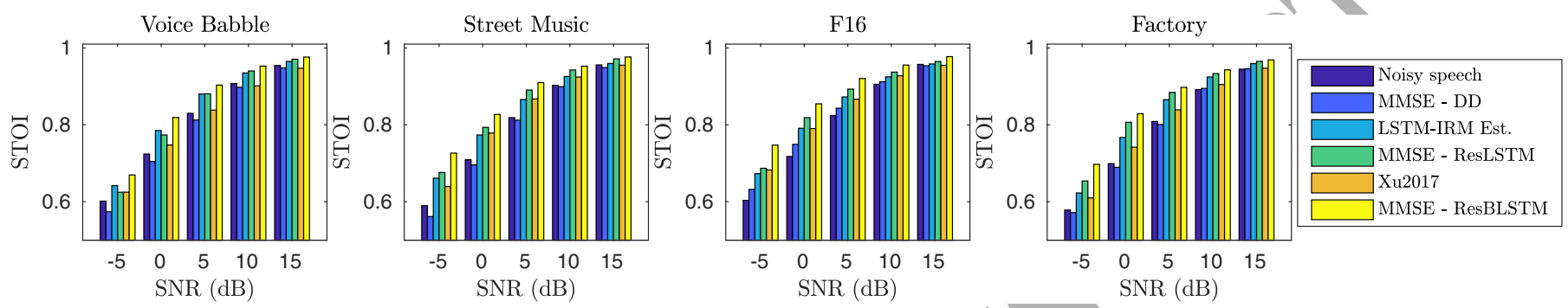

Figure 14: Objective intelligibility (STOI) scores for the MMSE-LSA estimator utilising the DD approach, the LSTM-IRM estimator, Xu2017, and the MMSE-LSA estimator utilising both the ResLSTM and ResBLSTM a priori SNR estimators. The tested conditions include real-world non-stationary (voice babble and street music) and coloured (F16 and factory) noise sources at multiple SNR levels.

section 6.3 , it can be deduced that most of the performance improvement gained by the MMSE-LSA estimator utilising the ResLSTM a priori SNR estimator over the LSTM-IRM estimator is due to the differing model and training strategy ${ }^{13}$, and not the training target. However, the opposite is likely true for Xu2017. The results from Subsection 6.3 indicate that most of the performance improvement gained by the MMSE-LSA estimator utilising the ResBLSTM a priori SNR estimator over Xu2017 is due to the training target, and not the model, training strategy, or post-processing.

\subsubsection{Subjective Quality Scores}

Subjective quality scores were obtained for the MMSELSA estimator utilising the DD approach, Xu2017, and the MMSE-LSA estimator utilising the ResBLSTM a priori SNR estimator. Details about the subjective testing procedure and the subjective test set are given in Subsection 5.7. Voice babble at an SNR level of $5 \mathrm{~dB}$ was the condition used for the subjective tests. The mean subjective preference (\%) for each of the speech enhancement methods is shown in Figure 15. It can be seen that the enhanced speech produced by the MMSE-LSA estimator utilising the ResBLSTM a priori SNR estimator (marked as MMSE-LSA (DL), where DL stands for deep learning) was preferred by listeners over Xu2017 enhanced speech.

\footnotetext{
${ }^{13}$ The LSTM-IRM estimator from [8] uses the quadratic loss function instead of the cross entropy loss function employed by the proposed a priori SNR estimators.
}

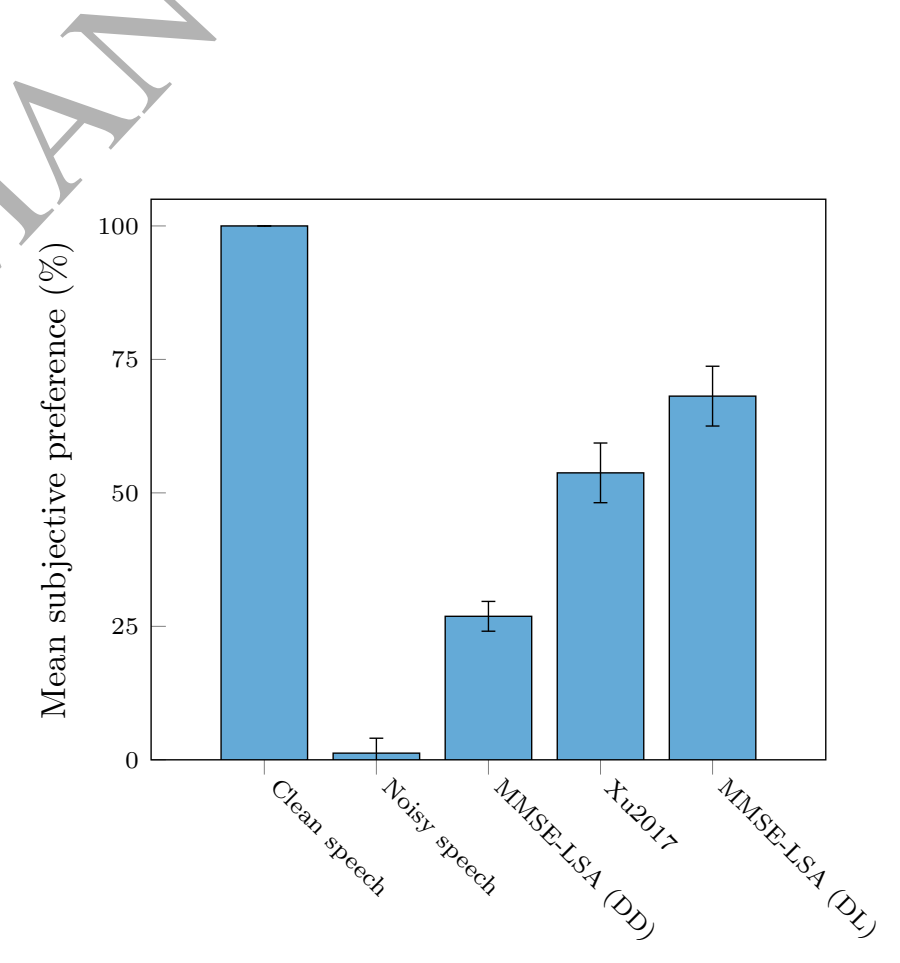

Figure 15: Mean subjective preference (\%) scores for the MMSE-LSA estimator utilising the DD approach (MMSE-LSA (DD)), Xu2017, and the MMSE-LSA estimator utilising the ResBLSTM a priori SNR estimator (MMSE-LSA (DL), where DL stands for deep learning). The subjective testing procedure is described in Subsection 5.7. Voice babble at an SNR level of $5 \mathrm{~dB}$ was the condition used for the subjective tests. 
(a) Clean speech

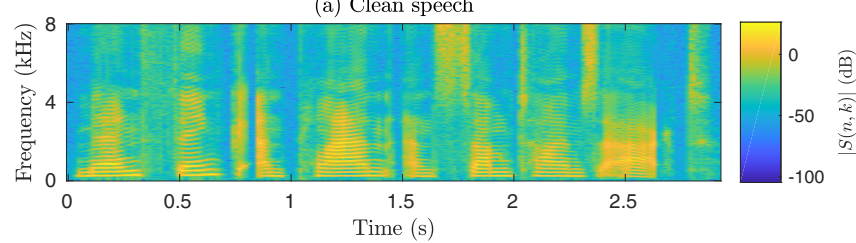

(b) Noisy speech; voice babble; $5 \mathrm{~dB}$

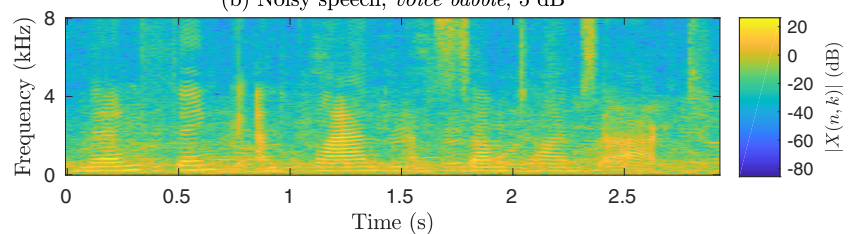

(c) MMSE-LSA estimator; DD approach

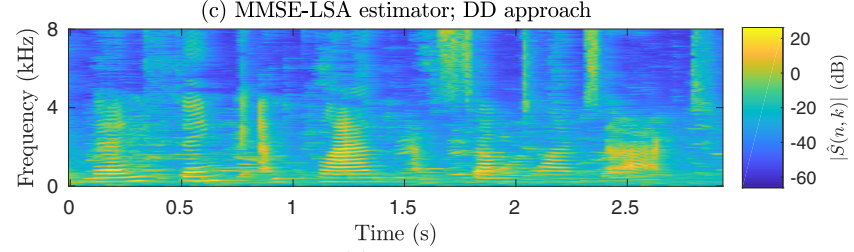

(d) $\mathrm{Xu} 2017$

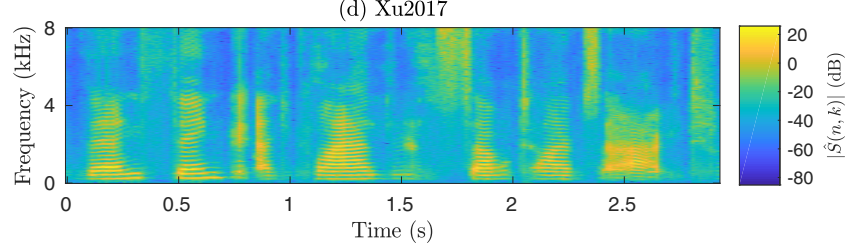

(e) MMSE-LSA estimator; ResBLSTM a priori SNR estimator

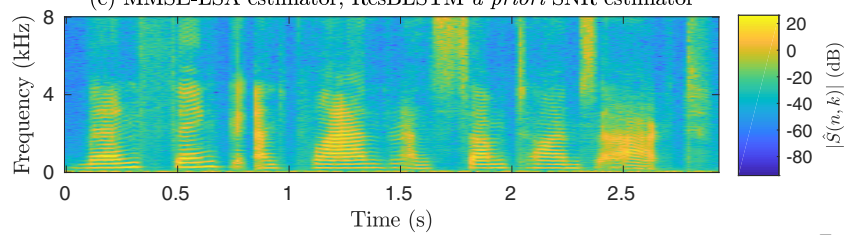

Figure 16: (a) Clean speech magnitude spectrogram of female $F F$ uttering sentence 32_10, "Men think and plan and sometimes act" from the test set. (b) A recording of voice babble mixed with (a) at an SNR level of $5 \mathrm{~dB}$. (c) Enhanced speech magnitude spectrogram produced by the MMSE-LSA estimator utilising the DD approach. (d) Enhanced speech magnitude spectrogram produced by Xu2017. (e) Enhanced speech magnitude spectrogram produced by the MMSELSA estimator utilising the ResBLSTM a priori SNR estimator.

\subsubsection{Enhanced Speech Spectrograms}

Shown in Figure 16 is the resultant enhanced speech magnitude spectrograms produced by the MMSE-LSA estimator utilising the DD approach, Xu2017, and the MMSE-LSA estimator utilising the ResBLSTM a priori SNR estimator. The clean and noisy speech magnitude spectrograms are shown in Figure 16 (a) and (b), respectively. The MMSE-LSA estimator utilising the ResBLSTM a priori SNR estimator was able to suppress most of the noise with little formant distortion (Figure 16 (e)). $\mathrm{Xu} 2017$ incorrectly suppressed some formant information (Figure $16(\mathrm{~d})$ ). The MMSE-LSA estimator utilising the DD approach demonstrated poor noise suppression (Figure $16(\mathrm{e}))$.

\subsection{Areas Requiring Further Investigation}

One factor that affects the performance of the MMSESTSA and MMSE-LSA estimators is the a posteriori SNR estimation accuracy. In this work, the a posteriori SNR estimate is computed using the a priori SNR estimate. Further performance gains may be achieved if deep learning methods are used to estimate the a posteriori SNR directly. Another area for investigation is the loss function. A recent trend has been to include the STOI measure in the loss function $[57,58]$. The speech enhancement performance of the proposed a priori SNR estimators may be improved if a perceptually motivated measure is integrated into the loss function.

\section{Conclusion}

Deep learning methods for MMSE approaches to speech enhancement are investigated in this work. A causal ResLSTM and a non-causal ResBLSTM are used here to accurately estimate the a priori SNR for the MMSE approaches. It was found that MMSE approaches utilising deep learning are able to produce enhanced speech that achieves higher quality and intelligibility scores than recent masking- and mapping-based deep learning approaches, for both real-world non-stationary and coloured noise soúrces. MMSE approaches utilising deep learning are currently being investigated for robust automatic speech recognition (ASR).

\section{References}

[1] Y. Ephraim, D. Malah, Speech enhancement using a minimummean square error short-time spectral amplitude estimator, IEEE Transactions on Acoustics, Speech, and Signal Processing 32 (1984) 1109-1121.

[2] Y. Ephraim, D. Malah, Speech enhancement using a minimum mean-square error log-spectral amplitude estimator, IEEE Transactions on Acoustics, Speech, and Signal Processing 33 (1985) 443-445.

[3] P. C. Loizou, Speech Enhancement: Theory and Practice, 2nd ed., CRC Press, Inc., Boca Raton, FL, USA, 2013.

[4] Z. Zhang, J. Geiger, J. Pohjalainen, A. E.-D. Mousa, W. Jin, B. Schuller, Deep learning for environmentally robust speech recognition: An overview of recent developments, ACM Trans. Intell. Syst. Technol. 9 (2018) 1-28.

[5] X. Lu, Y. Tsao, S. Matsuda, C. Hori, Speech enhancement based on deep denoising autoencoder, in: Proceedings Interspeech 2013, 2013, pp. 436-440.

[6] Y. Xu, J. Du, L. Dai, C. Lee, A regression approach to speech enhancement based on deep neural networks, IEEE/ACM Transactions on Audio, Speech, and Language Processing 23 (2015) 7-19.

[7] Y. Xu, J. Du, Z. Huang, L. Dai, C. Lee, Multi-objective learning and mask-based post-processing for deep neural network based speech enhancement, CoRR abs/1703.07172 (2017).

[8] J. Chen, D. Wang, Long short-term memory for speaker generalization in supervised speech separation, The Journal of the Acoustical Society of America 141 (2017) 4705-4714.

[9] O. Cappe, Elimination of the musical noise phenomenon with the Ephraim and Malah noise suppressor, IEEE Transactions on Speech and Audio Processing 2 (1994) 345-349. 
[10] C. Plapous, C. Marro, L. Mauuary, P. Scalart, A two-step noise reduction technique, in: 2004 IEEE International Conference on Acoustics, Speech, and Signal Processing, volume 1, 2004, pp. 289-292. doi:10.1109/ICASSP . 2004.1325979.

[11] C. Plapous, C. Marro, P. Scalart, Speech enhancement using harmonic regeneration, in: Proceedings. (ICASSP '05). IEEE International Conference on Acoustics, Speech, and Signal Processing, volume 1, 2005, pp. 157-160. doi:10.1109/ICASSP. 2005.1415074.

[12] C. Breithaupt, T. Gerkmann, R. Martin, A novel a priori SNR estimation approach based on selective cepstro-temporal smoothing, in: 2008 IEEE International Conference on Acoustics, Speech and Signal Processing, 2008, pp. 4897-4900. doi:10. 1109/ICASSP . 2008.4518755.

[13] J. Kim, M. El-Khamy, J. Lee, Residual LSTM: design of a deep recurrent architecture for distant speech recognition, CoRR abs/1701.03360 (2017).

[14] M. Schuster, K. K. Paliwal, Bidirectional recurrent neural networks, IEEE Transactions on Signal Processing 45 (1997) 26732681.

[15] Y. Xia, R. Stern, A priori SNR estimation based on a recurrent neural network for robust speech enhancement, in: Proc. Interspeech 2018, 2018, pp. 3274-3278. doi:10.21437/Interspeech. 2018-2423.

[16] J. Allen, Short term spectral analysis, synthesis, and modification by discrete Fourier transform, IEEE Transactions on Acoustics, Speech, and Signal Processing 25 (1977) 235-238.

[17] J. B. Allen, L. R. Rabiner, A unified approach to short-time Fourier analysis and synthesis, Proceedings of the IEEE 65 (1977) 1558-1564.

[18] P. Vary, R. Martin, Digital Speech Transmission: Enhancement, Coding and Error Concealment, John Wiley \& Sons, Inc., USA, 2006.

[19] D. Griffin, J. Lim, Signal estimation from modified short-time Fourier transform, IEEE Transactions on Acoustics, Speech, and Signal Processing 32 (1984) 236-243.

[20] R. Crochiere, A weighted overlap-add method of short-time Fourier analysis/synthesis, IEEE Transactions on Acoustics, Speech, and Signal Processing 28 (1980) 99-102.

[21] C. Plapous, C. Marro, P. Scalart, Improved signal-to-noise ratio estimation for speech enhancement, IEEE Transactions on Audio, Speech, and Language Processing 14 (2006) 2098-2108.

[22] J. S. Lim, A. V. Oppenheim, Enhancement and bandwidth compression of noisy speech, Proceedings of the IEEE 67 (1979) 1586-1604.

[23] K. He, X. Zhang, S. Ren, J. Sun, Deep residual learning for image recognition, CoRR abs/1512.03385 (2015).

[24] K. He, X. Zhang, S. Ren, J. Sun, Identity mappings in deep residual networks, CoRR abs/1603.05027 (2016).

[25] S. Hochreiter, J. Schmidhuber, Long short-term memory, Neural Computation 9 (1997) 1735-1780.

[26] F. A. Gers, J. Schmidhuber, F. Cummins, Learning to forget: continual prediction with LSTM, in: Ninth International Conference on Artificial Neural Networks (ICANN), volume 2, 1999 , pp. 850-855 vol.2. doi:10.1049/cp: 19991218.

[27] Y. Wu, M. Schuster, Z. Chen, Q. V. Le, M. Norouzi, W. Macherey, M. Krikun, Y. Cao, Q. Gao, K. Macherey, J. Klingner, A. Shah, M. Johnson, X. Liu, L. Kaiser, S. Gouws, Y. Kato, T. Kudo, H. Kazawa, K. Stevens, G. Kurian, N. Patil, W. Wang, C.Young, J. Smith, J. Riesa, A. Rudnick, O. Vinyals, G. Corrado, M. Hughes, J. Dean, Google's neural machine translation system: Bridging the gap between human and machine translation, CoRR abs/1609.08144 (2016).

[28] V. Nair, G. E. Hinton, Rectified linear units improve restricted boltzmann machines, in: Proceedings of the 27th International Conference on International Conference on Machine Learning, ICML'10, Omnipress, USA, 2010, pp. 807-814.

[29] J. L. Ba, J. R. Kiros, G. E. Hinton, Layer normalization, arXiv preprint arXiv:1607.06450 (2016).

[30] J. Hanson, K. Paliwal, T. Litfin, Y. Yang, Y. Zhou, Accurate prediction of protein contact maps by coupling residual two- dimensional bidirectional long short-term memory with convolutional neural networks, Bioinformatics 34 (2018) 4039-4045.

[31] A. Veit, M. J. Wilber, S. J. Belongie, Residual networks are exponential ensembles of relatively shallow networks, CoRR abs/1605.06431 (2016).

[32] H. Sak, A. Senior, F. Beaufays, Long short-term memory recurrent neural network architectures for large scale acoustic modeling, in: Proceedings Interspeech 2014, 2014, pp. 338-342.

[33] J. W. Picone, Signal modeling techniques in speech recognition, Proceedings of the IEEE 81 (1993) 1215-1247.

[34] X. Huang, A. Acero, H.-W. Hon, Spoken Language Processing: A Guide to Theory, Algorithm, and System Development, 1st ed., Prentice Hall PTR, Upper Saddle River, NJ, USA, 2001.

[35] K. Paliwal, K. Wojcicki, Effect of analysis window duration on speech intelligibility, IEEE Signal Processing Letters 15 (2008) $785-788$.

[36] T. Gerkmann, R. C. Hendriks, Unbiased MMSE-based noise power estimation with low complexity and low tracking delay, IEEE Transactions on Audio, Speech, and Language Processing 20 (2012) 1383-1393.

[37] V. Panayotov, G. Chen, D. Povey, S. Khudanpur, Librispeech: An ASR corpus based on public domain audio books, in: 2015 IEEE International Conference on Acoustics, Speech and Signal Processing (ICASSP), 2015, pp. 5206-5210. doi:10.1109/ ICASSP . 2015.7178964.

[38] C. Veaux, J. Yamagishi, K. MacDonald, et al., CSTR VCTK corpus: English multi-speaker corpus for CSTR voice cloning toolkit, University of Edinburgh. The Centre for Speech Technology Research (CSTR) (2017).

[39] J. S. Garofolo, L. F. Lamel, W. M. Fisher, J. G. Fiscus, D. S. Pallett, DARPA TIMIT acoustic-phonetic continuous speech corpus CD-ROM. NIST speech disc 1-1.1, NASA STI/Recon Techrical Report N 93 (1993).

[40] D. B. Dean, S. Sridharan, R. J. Vogt, M. W. Mason, The QUT-NOISE-TIMIT corpus for the evaluation of voice activity detection algorithms, in: Proceedings Interspeech 2010, 2010, pp. 3110-3113.

[41] G. Hu, 100 nonspeech environmental sounds, The Ohio State University, Department of Computer Science and Engineering (2004).

[42] F. Saki, A. Sehgal, I. Panahi, N. Kehtarnavaz, Smartphonebased real-time classification of noise signals using subband features and random forest classifier, in: 2016 IEEE International Conference on Acoustics, Speech and Signal Processing (ICASSP), 2016, pp. 2204-2208. doi:10.1109/ICASSP. 2016. 7472068 .

[43] F. Saki, N. Kehtarnavaz, Automatic switching between noise classification and speech enhancement for hearing aid devices, in: 2016 38th Annual International Conference of the IEEE Engineering in Medicine and Biology Society (EMBC), 2016, pp. 736-739. doi:10.1109/EMBC.2016.7590807.

[44] D. Snyder, G. Chen, D. Povey, MUSAN: A music, speech, and noise corpus, CoRR abs/1510.08484 (2015).

[45] D. P. Kingma, J. Ba, Adam: A method for stochastic optimization, CoRR abs/1412.6980 (2014).

[46] H. J. Steeneken, F. W. Geurtsen, Description of the RSG-10 noise database, Report IZF 1988-3, TNO Institute for Perception, Soesterberg, The Netherlands (1988).

[47] J. Salamon, C. Jacoby, J. P. Bello, A dataset and taxonomy for urban sound research, in: Proceedings of the 22nd ACM International Conference on Multimedia, MM '14, ACM, New York, NY, USA, 2014, pp. 1041-1044. doi:10.1145/2647868. 2655045.

[48] P. Kabal, TSP speech database, McGill University, Database Version (2002).

[49] K. K. Paliwal, B. S. Atal, Efficient vector quantization of LPC parameters at 24 bits/frame, in: Proceedings ICASSP 91: 1991 International Conference on Acoustics, Speech, and Signal Processing, 1991, pp. 661-664 vol. 1. doi:10.1109/ICASSP.1991. 150426.

[50] ITU-T Recommendation P.800.1, Mean opinion score (MOS) 
terminology, 2006.

[51] ITU-T Recommendation P.862.2, Wideband extension to recommendation P.862 for the assessment of wideband telephone networks and speech codecs, 2007.

[52] C. H. Taal, R. C. Hendriks, R. Heusdens, J. Jensen, A short-time objective intelligibility measure for time-frequency weighted noisy speech, in: 2010 IEEE International Conference on Acoustics, Speech and Signal Processing, 2010, pp. 42144217. doi:10.1109/ICASSP . 2010.5495701.

[53] C. H. Taal, R. C. Hendriks, R. Heusdens, J. Jensen, An algorithm for intelligibility prediction of time-frequency weighted noisy speech, IEEE Transactions on Audio, Speech, and Language Processing 19 (2011) 2125-2136.

[54] S. So, K. K. Paliwal, Modulation-domain Kalman filtering for single-channel speech enhancement, Speech Communication 53 (2011) $818-829$.

[55] Y. Wang, A. Narayanan, D. Wang, On training targets for supervised speech separation, IEEE/ACM Transactions on Audio, Speech, and Language Processing 22 (2014) 1849-1858.

[56] Y. Zhao, D. Wang, I. Merks, T. Zhang, DNN-based enhancement of noisy and reverberant speech, in: 2016 IEEE International Conference on Acoustics, Speech and Signal Processing (ICASSP), 2016, pp. 6525-6529. doi:10.1109/ICASSP. 2016. 7472934.

[57] S. Fu, T. Wang, Y. Tsao, X. Lu, H. Kawai, End-to-end waveform utterance enhancement for direct evaluation metrics optimization by fully convolutional neural networks, IEEE/ACM Transactions on Audio, Speech, and Language Processing 26 (2018) 1570-1584.

[58] Y. Zhao, B. Xu, R. Giri, T. Zhang, Perceptually guided speech enhancement using deep neural networks, in: 2018 IEEE International Conference on Acoustics, Speech and Signal Processing (ICASSP), 2018, pp. 5074-5078. doi:10.1109/ICASSP. 2018. 8462593. 


\section{Accepted Manuscript}

Deep Learning for Minimum Mean-Square Error Approaches to Speech Enhancement

Aaron Nicolson, Kuldip K. Paliwal

PII:

DOI: S0167-6393(18)30430-8

Reference: https://doi.org/10.1016/j.specom.2019.06.002 SPECOM 2649

To appear in:

\section{Speech Communication}

Received date: Revised date:

Accepted date:

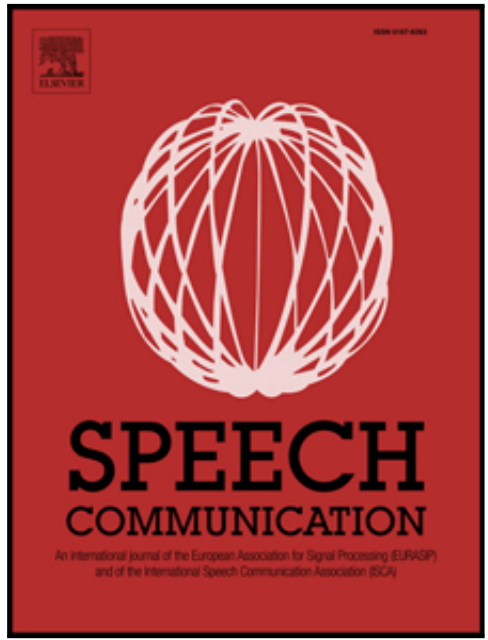

Please cite this article as: Aaron Nicolson, Kuldip K. Paliwal, Deep Learning for Minimum Mean-Square Error Approaches to Speech Enhancement, Speech Communication (2019), doi: https://doi.org/10.1016/j.specom.2019.06.002

This is a PDF file of an unedited manuscript that has been accepted for publication. As a service to our customers we are providing this early version of the manuscript. The manuscript will undergo copyediting, typesetting, and review of the resulting proof before it is published in its final form. Please note that during the production process errors may be discovered which could affect the content, and all legal disclaimers that apply to the journal pertain. 